Original Research Paper

\title{
Evaluation of the Texas Feed Yard Workforce: Survey of Stockperson Attitudes and Perceptions towards Euthanasia, Animal Care and Employee Value
}

\author{
${ }^{1}$ Emily E. Ridge, ${ }^{2}$ Ron Gill and ${ }^{1}$ Courtney L. Daigle \\ ${ }^{1}$ Texas A\&M University Department of Animal Science, College Station Texas 77843, USA \\ ${ }^{2}$ Texas A\&M AgriLife Extenstion, College Station Texas 77843, USA
}

Article history

Received: 22-02-2019

Revised: 11-05-2019

Accepted: 27-05-2019

Corresponding author: Courtney L. Daigle

2471 TAMU, Room 212B

College Station TX 77843 USA

Tel: 979-862-9171

Email: cdaigle@tamu.edu

\begin{abstract}
A survey was administered to Texas cattle feedyard employees $(\mathrm{n}=111)$ from 31 different operations measuring stockperson perception, job satisfaction and socioeconomic status using Likert statements and multiple-choice questions. Differences among employment roles (manager, pen rider, processor (those who vaccinate and implant incoming calves), doctor (non-veterinarians)) were evaluated using a Kruskal-Wallis test followed by a Multiple Comparison procedure. Managers strongly agreed with the statement "beef cattle are not dirty" more than doctors $(P=0.03)$ and that "cattle behavior is affected by the way we treat them" than pen riders $(P=0.002)$ or processors $(P=0.01)$. Managers were less likely than doctors, pen riders and processors to believe they have too many cattle to look after $(P=0.05, P=0.006$ and $P=0.01$, respectively $)$. Pen riders reported less confidence in performing euthanasia than doctors $(P=0.02)$ and managers $(P=0.02)$ and, along with processors, agreed that cattle were not always euthanized in a timely manner $(P=0.02$ and $P=0.02$, respectively). While all roles viewed Holstein cattle unfavorably $(P<$ $0.001)$, processors viewed them more positively than pen riders $(P=0.05)$ and managers $(P=0.001)$. Socioeconomic results showed that Texas feedyards have a dedicated, passionate work force, with $43 \%$ of participants having worked in the industry for $9+$ years and $49 \%$ of participants working at cattle feedyards because they enjoy working with animals. Unfortunately, it was evident that stock people are underpaid (57\% of participants making between $\$ 10-15 / \mathrm{hr}$ ) and overworked (76\% of participants working $50+$ hrs/week). Survey responses identified critical role-dependent knowledge gaps and biases. A disconnect was observed among compensation, workload and the duration of time stock people spend interacting with cattle. Increasing industry investment in feedyard employees and providing breed-specific and employee role-specific education may promote an encouraging workplace that ensures feedlot cattle experience good welfare.
\end{abstract}

Keywords: Stockperson, Job Satisfaction, Animal Perception, Euthanasia

\section{Introduction}

Awareness by livestock producers and consumers regarding the importance of the human-animal interaction (HAI) to livestock welfare is increasing as an overwhelming body of research suggests that stockperson behavior has the ability to directly impact animal behavior and health (Hemsworth and Coleman, 2011). Modern modes of animal agriculture demand that stock people interact frequently with animals to provide daily care and maintenance. As regular interactions with humans are a critical component of dairy and swine production, the dairy and swine industries have quantified how stockperson personality, attitude, perceptions and behavior directly affects the productivity, growth and welfare of livestock (Hemsworth et al., 1993). However, the impact of the HAI has not been objectively evaluated in the feeder cattle industry. Tucker et al., (2015) summarizes that much of the 
previous research evaluating beef cattle has emphasized nutritional efficiency, reproductive success, and the consequences of the HAI with regard to painful procedures (e.g., castration, dehorning, branding). Little research has explored the impact of group-housed feeder cattle caretakers (e.g., pen riders, processors, doctoring crews, feed truck drivers, and feed yard managers) on cattle welfare (Rouse, 2003). Husbandry guidelines have been developed for feeder cattle, yet these guidelines focus on housing and health management and do not explicitly outline expectations regarding the HAI (BCO, 2015).

A very small proportion of the United States population is engaged in animal agriculture, with less than 300,000 Americans working with "farm, ranch and aquaculture animals" (USBS 2018a). As a consequence, many of the individuals that constitute the current and future generations of stock people may not have the same inherent knowledge base as previous generations regarding animal husbandry and handling because they grew up in an urban environment, rather than in an agriculturally-centered community. This requires the current population of stock people to quantify and record their husbandry practices for use by the current and future generations and this need for documentation includes evaluating the impact of the HAI on cattle welfare. Therefore, to develop guidelines that can be transferred to the next generation of stock people, an objective understanding of the HAI between stock people and beef cattle is imperative.

Attitude based psychology suggests "as a general rule, we tend to behave in favorable ways with respect to things and people we like and display unfavorable behaviors towards things and people we dislike and, barring unforeseen events, we translate our plans into actions" (Janzen, 1991). This theory implies that a stockperson's attitude, how much they like or dislike things, forms the perceptions they hold regarding job satisfaction and their work environment. Further, the Theory of Planned Behavior (Janzen, 1991) can apply to the stockperson-livestock relationship. The HAI can become easily impaired if the stockperson holds negative attitudes towards animals. If a stockperson does not like the animals in their care, they can translate this dislike into negative interactions with the animal. Previous research (Waiblinger et al., 2002) suggests that affirmative stockperson attitudes were positively associated with positive handing experiences and positive attitudes have been connected to stock people that use gentle touching techniques and calm vocalizations to move animals (Hemsworth and Coleman, 2011). Therefore, positive stockperson attitude influences the prevalence of positive behaviors towards the animals in their care.
The relationship between stock people and livestock is dynamic and reciprocal (Carlstead et al.,, 2019). Effective animal care and optimal animal productivity can be most easily achieved when there is a positive HAI on-farm. Stockperson attitudes and perceptions expressed as physical behaviors directly impacts the quality of HAI. In turn, any negative HAI experiences can produce a fear response by the animal. Fear can negatively affect livestock performance and welfare (Hemsworth and Coleman, 2011). Animals that display a strong fear response towards humans have been associated with reduced productivity, including decreased feed efficiency and egg laying rates in poultry (Hemsworth and Barnett, 1989) and lower average milk yield in dairy cows (Waiblinger et al., 2002). Reducing the fear of humans by livestock is essential to ensure proper animal welfare and high rates of productivity. One mechanism to reduce fear induced by a negative HAI is to adjust the perceptions of stock people.

With more information on beef cattle stockperson attitudes and behaviors, the industry can begin to develop effective training interventions and workplace enrichments for livestock operations. Providing cattle with a welfare-positive environment requires stock people to possess a wide range of knowledge regarding animal handing, treatment and feeding-all of which require education, training and skill development. Providing training to stock people through behavioral intervention and physical training can improve the attitude of the stockperson and increase positive human-animal interactions (Hemsworth et al., 2002). However, the educational and training needs must be identified prior to material generation and implementation.

In addition to improving the HAI, training is also beneficial for the well-being of the stockperson. Occupational psychology research observed that employees valued training, understood that training a necessary part of their jobs and that training benefited themselves, their co-workers and improved the workplace environment (Schmidt, 2007). Moreover, workplace training positively influenced employee attitudes and commitment (Schmidt, 2007). Swine operations that provided training interventions to their stock people experienced a $52 \%$ higher retention rate in those that received training compared to those that did not (Hemsworth et al., 1994). Training has also been effective in increasing empathy in stock people, a critical component of a good HAI (Coleman and Hemsworth, 2014). Increased job commitment is mutually beneficial to the stockperson, the employer, and the animal.

Providing training to stock people communicates that employers are expressing care for both the animals and their employees and can have a positive impact on stockperson attitude regarding the workplace and job satisfaction. Employee perception of job satisfaction can subsequently lead to the growth of interpersonal relationships among coworkers and management (Sari and Judge, 2004). Which can have positive ramifications for the employee unit and 
workplace culture. Therefore, investing in employees' satisfaction can not only have animal welfare impacts but also stockperson well-being and long-term sustainability for animal agriculture (Daigle and Ridge, 2018).

This study was designed to collect preliminary data on the population of stock people working in Texas beef cattle feed yards. A survey was administered to gather empirical evidence on the current job satisfaction, animal perceptions and socio-economic status of feed yard stock people. Information from this survey will facilitate the development of husbandry guidelines and management strategies designed to educate, train and retain feed yard employees in order to improve animal welfare and increase industry longevity.

\section{Materials and Methods}

A survey was administered across three days as part of an annual Texas Cattle Feeders Association training session conducted in the Texas Panhandle. Feed yard employees $(n=111)$ from 31 different feed yards that attended the training session completed the survey. Survey questions addressed topics that included employee perception of livestock, job satisfaction and socioeconomic variables. The research project and survey questions were approved by the Texas A\&M University Institutional Review Board (IRB2017-0936M).

Table 1: Likert statement questions and percentage of responses by likert group for all roles

\begin{tabular}{|c|c|c|c|c|c|}
\hline Statement & $\begin{array}{l}\text { Strongly } \\
\text { Agree }\end{array}$ & $\begin{array}{l}\text { Somewhat } \\
\text { Agree }\end{array}$ & $\begin{array}{l}\text { Somewhat } \\
\text { disagree }\end{array}$ & $\begin{array}{l}\text { Strongly } \\
\text { disagree }\end{array}$ & $\begin{array}{l}\text { I don't } \\
\text { know }\end{array}$ \\
\hline \multicolumn{6}{|l|}{ Empathy } \\
\hline I feel uncomfortable when my coworker hits cattle & 89 & 90 & 00 & 00 & 20 \\
\hline I feel uncomfortable when my coworker inappropriately uses an electric prod & 28 & 18 & 29 & 23 & 20 \\
\hline I feel uncomfortable when my coworker yells at cattle & 38 & 37 & 14 & 90 & 30 \\
\hline I get upset more than other people when I see cattle in pain & 54 & 26 & 90 & 50 & 60 \\
\hline \multicolumn{6}{|l|}{ Perception of beef cattle } \\
\hline I like working with beef cattle & 01 & 30 & 26 & 68 & 10 \\
\hline Beef cattle are not dirty & 67 & 10 & 50 & 18 & 10 \\
\hline Beef cattle are smart & 11 & 12 & 10 & 56 & 12 \\
\hline Beef cattle are individuals with their own personalities & 13 & 16 & 21 & 37 & 13 \\
\hline Beef cattle are not tough & 25 & 11 & 15 & 38 & 12 \\
\hline Beef cattle behavior is affected by the way we treat them & 31 & 24 & 17 & 13 & 16 \\
\hline \multicolumn{6}{|l|}{ Perception of dairy cattle } \\
\hline I like working with Holsteins & 21 & 17 & 25 & 23 & 15 \\
\hline Holsteins are not dirty & 37 & 16 & 12 & 24 & 11 \\
\hline Holsteins are smart & 76 & 20 & 10 & 20 & 10 \\
\hline Holsteins are individuals with their own personalities & 66 & 17 & 90 & 40 & 40 \\
\hline Holsteins are not tough & 67 & 19 & 60 & 40 & 40 \\
\hline Holstein behavior is affected by the way we treat them & 46 & 24 & 14 & 12 & 40 \\
\hline \multicolumn{6}{|l|}{ Job satisfaction } \\
\hline I like my job & 39 & 18 & 25 & 16 & 30 \\
\hline I like coming to work & 58 & 12 & 23 & 80 & 00 \\
\hline I have enough time during my shift to do my job well & 59 & 24 & 12 & 50 & 00 \\
\hline I do not have too many cattle to look after & 70 & 23 & 40 & 20 & 10 \\
\hline I don't feel pressure to do my job quickly & 52 & 30 & 70 & 70 & 50 \\
\hline \multicolumn{6}{|l|}{ Trust and communication with co-workers and management } \\
\hline I find it easy to communicate with my boss & 52 & 27 & 90 & 40 & 80 \\
\hline I find it easy to communicate with my coworkers & 48 & 17 & 17 & 80 & 10 \\
\hline I feel confident asking for help when solving a problem at work & 43 & 24 & 16 & 11 & 70 \\
\hline I trust my coworkers to provide good care to cattle when I am away from work & k 19 & 19 & 23 & 35 & 50 \\
\hline My coworkers are as good as I am at caring for cattle & 34 & 33 & 15 & 11 & 60 \\
\hline \multicolumn{6}{|l|}{ Perception of euthanasia } \\
\hline I feel confident that I know when cattle need to be euthanized & 34 & 20 & 21 & 17 & 80 \\
\hline I think about cattle feelings when I euthanize it & 61 & 32 & 20 & 30 & 30 \\
\hline If I had the choice I prefer I euthanize cattle rather than someone else & 68 & 13 & 90 & 60 & 30 \\
\hline $\begin{array}{l}\text { My coworkers do not wait longer than they should before euthanizing cattle } \\
\text { Job knowledge }\end{array}$ & 57 & 33 & 50 & 30 & 20 \\
\hline The best place to keep an electric prod is away from me & 79 & 12 & 20 & 20 & 50 \\
\hline I am good at handling cattle & 75 & 12 & 60 & 50 & 20 \\
\hline I have enough knowledge to know what to do with sick or injured cattle & 16 & 11 & 20 & 43 & 90 \\
\hline It is easy to identify sick or injured cattle & 34 & 16 & 13 & 30 & 80 \\
\hline I feel confident providing medical treatment to sick cattle & 33 & 11 & 23 & 19 & 14 \\
\hline
\end{tabular}


Emily. E. Ridge et al. / American Journal of Animal and Veterinary Sciences 2019, 14 (2): 139.150 DOI: $10.3844 /$ ajavsp.2019.139.150

Table 2: Percentage of participant responses for each socio-economic related question for all roles

\section{Question}

Results (\%)

How long have you worked at a feedyard in your lifetime?

Less than 1 year

1 to 3 years

16

3 to 6 years

6 to 9 years

10

$9+$ years

How long have you worked at your current feedyard?

Less than 6 months

6 months to 1 year

1 to 2 years

2 to 3 years

$3+$ years

Why did you choose to work in a beef cattle feedyard?

Needed a job

Family business

Pay

Enjoy working with animals

Why do you stay working at a beef cattle feedyard?

Career

Needed a job

Family business

Pay

Enjoy working with animals

Yes

How many hours per week do you work?

Less than 40 hours

40 to 50 hours

50 to 60 hours

60 to 70 hours

$70+$ hours

What is your hourly wage? (per hour)

$<\$ 10$

$\$ 10$ to $\$ 15$

$\$ 15$ to $\$ 20$

$\$ 20$ to $\$ 25$

$>\$ 25$

What is your highest level of education?

Less than high school

High school diploma/GED

Trade school

Some college

College degree

Are you a veteran?

Yes

I speak fluently in...

English only

50

Spanish only 
Table 2: Continue

Are you the only member of your family that provides household income? Yes

No

How old are you?

Under 18

18 to 30

Participants were asked to respond to Likert statements using a 5-point Likert scale, ranging from "strongly disagree" to "strongly agree" with an "I don't know option". Various questions were also administered using a multiple choice format. Survey responses were collected using iClicker remote technology. Prior to survey administration, participants were oriented to the iClicker technology and were able to familiarize themselves with the remote using a test question.

Participants remained anonymous as the iClicker remotes were provided to the participants randomly and the remotes were not connected to the participants' identity. Survey questions were projected onto a whiteboard to be visually available for reading in both Spanish and English. Survey questions were read aloud in English and a Spanish interpreter provided verbal translations of the survey to the participants that spoke exclusively Spanish. The survey consisted of 50 questions total: 35 Likert scale questions (Table 1) designed to measure animal perception and job training and satisfaction and 15 multiple-choice questions (Table 2) addressing workforce demographics and individual socioeconomic status. The survey took approximately $30 \mathrm{~min}$ to administer and was administered to 6 separate groups ranging from 6 to 31 participants. Participants were questioned in smaller groups to accommodate the other activities occurring as part of the on-farm training event.

Stock people participants were asked to identify their employment role as either a processor, pen rider, doctor, or manager. Processors are identified as individuals who are responsible administering growth implants and vaccinations to incoming calves as well as sorting and re-implanting fattened cattle. These employees interact directly with each animal that arrives at the feed yard and these interactions are typically while the human is on foot and occurs at a handling facility. Pen riders are characterized as employees who are responsible for the daily health and care of feedlot cattle. Pen riders will visually examine cattle in their home pen from either on foot or while riding horseback on a daily basis. Pen riders tend to work closely with doctors, as doctors are responsible for the medical treatment of any animals which were removed from the main herd by the pen riders due to illness. The doctoring crew will primarily interact with cattle at the hospital facilities and these interactions typically occur while the human is on foot. Doctors also perform necropsies and euthanasia. Doctors are not typically college trained (i.e., not veterinarians or veterinarian technicians) but rather employees who have been trained on-the-job by consulting veterinarians to implement veterinarian approved protocols for treating cattle illnesses and injuries. Feedyard managers supervise pen riders, doctors, and processors, control animal feeding, and organize incoming and outgoing cattle shipments. Their interactions with cattle are limited compared to processors, pen riders and doctors; however, they may interact with cattle on foot, from horseback, or from a truck.

\section{Animal Perception (20 Statements)}

Stock people were asked about their general beliefs towards animals, specifically beef cattle and dairy cattle (Table 1). Statements evaluated how much the employee enjoyed working with each type of animal (beef cattle and dairy cattle) and how strongly the stockperson believed that each type of animal possessed emotions, individual personality, intelligence, constitution and whether the animal's behavior can be influenced by human behavior. Stock people were also presented statements regarding onfarm animal euthanasia.

\section{Job Training and Satisfaction (15 Statements)}

Participants were asked to provide their perception of job satisfaction, workplace communication and coworker trust (Table 1). Statements included topics such as their perceived job knowledge, ability to identify and treat sick animals and how to handle cattle. They were asked to respond about how they feel towards their coworker's behavior and to evaluate how well they communicate with their boss.

\section{Workforce Demographics and Socio-Economic status (15 questions)}

Participants were asked questions regarding employment history, wage, age, and education level using a multiple-choice format (Table 2). Topics included the motivation for employment at the feed yard, experience and preferences about their job. 


\section{Statistical Analysis}

Survey questions we evaluated as both individual questions and in Likert groups. Likert groups consisted of "empathy" (4 questions), "perception of beef cattle" (6 questions), "perception of dairy cattle" (6 questions), "job satisfaction" (5 questions), "trust and communication" (5 questions), "knowledge" (5 questions) and "euthanasia" (4 questions).

Likert scale questions were given values of 0 to 5 , with "strongly agree" having the highest numerical value, "strongly disagree" having a numerical value of 1 and "I don't know" scoring a zero. High Likert values represent a "positive perception" of the Likert statement, in which the participant is agreeing that they enjoy animal and their jobs and believe they possess adequate job knowledge. Low Likert values are associated with a "negative perception", in which the participant is disagreeing that they enjoy animals and their workplace.

Individual Likert statements results were analyzed for perception differences by role (pen rider, processors, doctors and management) using a Kruskal-Wallis procedure. A pairwise Wilcoxon test was applied to any statement in which roles present different perceptions to determine the directionality and the role(s) that differed significantly from other roles. A multivariate nonparametric test was performed to determine the perception differences between beef and dairy (Holsteins being fed for meat production in a feed yard, not Holsteins residing in a dairy) breeds regardless of role. Differences regarding perception of Likert groups, regardless of role, was evaluated using a Multiple Response Permutation Procedure (MRPP). All procedures were performed using R Statistical Software (2018).

\section{Results}

\section{Perception Differences by Role}

Managers were more likely to agree than doctors $(P=$ 0.03 ) that "beef cattle are not dirty". Managers also more strongly agreed that "beef cattle behavior is affected by the way we treat them" than pen riders $(P=0.002)$ and processors $(P=0.01)$. All roles displayed a strong dislike for working with dairy cattle $(P<0.0001)$. Among roles, processors tolerated working with dairy cattle the most compared to managers $(P=0.006)$ and pen riders $(P=0.005)$.

Pen riders felt more uncomfortable performing euthanasia than doctors $(P=0.02)$ and managers $(P=0.02)$. Pen riders reported discomfort with euthanasia timing and agreed that euthanasia is not performed in a timely manner while management does believe euthanasia occurs when it should $(P=0.02)$. Along with pen riders, processors also disagreed with management in believing euthanasia does not occur in a timely manner on farm $(P=0.04)$.

Role differed on job satisfaction Likert statements the least, with all roles displaying high levels of job satisfaction. Managers agreed that "they don't have too many cattle to look after", while doctors, pen riders and processors were less likely to agree with this statement ( $P=0.05, P=0.006$ and $P=0.01$, respectively).

None of the roles differed within the "knowledge", "empathy", or "trust and communication" Likert group statements. Participants displayed confidence regarding the ability to perform their jobs correctly, as shown by the high proportion of "strongly agree" answers for the "knowledge" statements (Table 1).

\section{Perception Differences Among all Roles}

The median Likert scores of the roles in the Likert groups (empathy, beef, dairy, job satisfaction, trust and communication, euthanasia and knowledge) were evaluated (Table 3). Roles differed in their view of overall perception of beef cattle $(P=0.005)$, where doctors and pen riders had the most positive perception of beef cattle. The Likert group "job satisfaction" differed by role, were processors had the lowest level of job satisfaction $(P=0.04)$. Lastly, euthanasia Likert questions also differed by role $(P=0.01)$. Pen riders and processors had median values of 2 , signaling they lacked confidence regarding life-ending choices.

\section{Demographic Characteristics}

Among the surveyed stock people, $36 \%$ were pen riders, $16 \%$ were doctors, $14 \%$ were processors, and $31 \%$ of participants were managers. Feed truck drivers accounted for $4 \%$ of participants but due to a low sample size, their answers were eliminated from the study. Demographic characteristics are summarized in Table 2. Across all roles, $76 \%$ of participants worked $50+$ hours per week while the majority of participants $(57 \%)$ made \$10-15 per hour. Most survey participants were aged 1840 and $53 \%$ of all survey participants had worked in the beef cattle feed yard industry for $6+$ years.

There was a wide range in education, age and language fluency among feed yard employees. Across all roles, $25 \%$ of employees possessed a college degree while $54 \%$ possessed a high school diploma or less. These results highlighted a generational gap, where $41 \%$ of participants were under 30 years of age while $39 \%$ were over 40 . Language fluency also varied, where $50 \%$ of those surveyed spoke only English, 14\% spoke only Spanish and $36 \%$ were bilingual in both Spanish and English.

Pen riders were asked two role-specific questions (Table 4). A majority of pen riders (84\%) were responsible for riding $30+$ pens per day. Many Texas feed yards house 150-200 animals per pen, suggesting that the average Texas pen rider is likely visually evaluating the health of approximately 6,000 animals per day. Pen riders were 
asked which strategy ("wave" or "section") was used to ride pens while conducting visual health observations. The "wave" method was used by $32 \%$ of surveyed pen riders and is characterized by pen riders riding through the entire feed yard as a group, moving from one end of the yard to the other, resulting in pen riders becoming familiar with the entire landscape of the yard; however, they may not evaluate the same animals every day. Conversely, $52 \%$ of pen riders utilized the "section" method. The "section" method consists of the pen rider being assigned to a set of pens and riding these same pens every day, creating a scenario where a specific pen rider is responsible for a specific set of animals.

Table 3: Median Likert score for each statement, by role and corresponding p-value $(\mathrm{P}=0.05)$. Values ranged from 5 or "strongly agree" to 1 or "strongly disagree"

\begin{tabular}{|c|c|c|c|c|c|}
\hline Statement & Doctor & Pen rider & Processor & Manager & P-value \\
\hline Empathy & 4 & 4 & 4 & 4 & 0.60 \\
\hline I feel uncomfortable when my coworker hits cattle & 5 & 3 & 4 & 4 & 0.69 \\
\hline I feel uncomfortable when my coworker inappropriately uses an electric prod & 3 & 4 & 4.5 & 4 & 0.45 \\
\hline I feel uncomfortable when my coworker yells at cattle & 4 & 2 & 2 & 1 & 0.54 \\
\hline I get upset more than other people when I see cattle in pain & 4 & 4 & 4 & 4 & 0.93 \\
\hline Perception of beef cattle & $5^{\mathrm{a}}$ & $5^{\mathrm{a}}$ & $4^{\mathrm{b}}$ & $4^{\mathrm{b}}$ & 0.01 \\
\hline I like working with beef cattle & 5 & 5 & 5 & 5 & 0.08 \\
\hline Beef cattle are not dirty & $1.5^{\mathrm{a}}$ & $2^{\mathrm{ab}}$ & $2^{\mathrm{ab}}$ & $4^{\mathrm{b}}$ & 0.03 \\
\hline Beef cattle are smart & 4 & 4 & 4 & 4 & 0.17 \\
\hline Beef cattle are individuals with their own personalities & 5 & 5 & 5 & 4 & 0.54 \\
\hline Beef cattle are not tough & 1 & 1 & 1 & 1 & 0.68 \\
\hline Beef cattle behavior is affected by the way we treat them & $5^{\mathrm{ab}}$ & $5^{\mathrm{ab}}$ & $4^{\mathrm{b}}$ & $5^{\mathrm{a}}$ & 0.03 \\
\hline Perception of dairy cattle & 2 & 2 & 2 & 2 & 0.73 \\
\hline I like working with Holsteins & $1^{\mathrm{b}}$ & $1^{\mathrm{b}}$ & $1.5^{\mathrm{a}}$ & $1^{\mathrm{b}}$ & 0.01 \\
\hline Holsteins are not dirty & 1 & 1 & 2 & 2 & 0.58 \\
\hline Holsteins are smart & 1 & 1 & 1 & 1 & 0.92 \\
\hline Holsteins are individuals with their own personalities & 2 & 4 & 1.5 & 4 & 0.37 \\
\hline Holsteins are not tough & 2 & 2 & 1 & 2 & 0.61 \\
\hline Holstein behavior is affected by the way we treat them & 4 & 2 & 1.5 & 4 & 0.37 \\
\hline Job satisfaction & $5^{\mathrm{a}}$ & $5^{\mathrm{a}}$ & $4^{\mathrm{b}}$ & $5^{\mathrm{a}}$ & 0.04 \\
\hline I like my job & 5 & 5 & 5 & 5 & 0.20 \\
\hline I like coming to work & 5 & 5 & 4 & 5 & 0.66 \\
\hline I have enough time during my shift to do my job well & 5 & 5 & 5 & 5 & 0.22 \\
\hline I do not have too many cattle to look after & $4^{\mathrm{b}}$ & $4^{\mathrm{b}}$ & $4^{\mathrm{b}}$ & $5^{\mathrm{a}}$ & 0.02 \\
\hline I don't feel pressure to do my job quickly & 4 & 4 & 2 & 4 & 0.38 \\
\hline Trust and communication with co-workers and management & 5 & 5 & 5 & 5 & 0.51 \\
\hline I find it easy to communicate with my boss & 4.5 & 5 & 5 & 5 & 0.80 \\
\hline I find it easy to communicate with my coworkers & 5 & 5 & 4 & 4.5 & 0.17 \\
\hline I feel confident asking for help when solving a problem at work & 5 & 5 & 5 & 5 & 0.87 \\
\hline I trust my coworkers to provide good care to cattle when I am away from work & 4.5 & 5 & 4.5 & 4 & 0.55 \\
\hline My coworkers are as good as I am at caring for cattle & 5 & 4.5 & 4.5 & 4 & 0.81 \\
\hline Perception of euthanasia & $5^{\mathrm{a}}$ & $2^{\mathrm{b}}$ & $2^{\mathrm{b}}$ & $4^{\mathrm{a}}$ & 0.01 \\
\hline I feel confident that I know when cattle need to be euthanized & 5 & 5 & 5 & 5 & 0.12 \\
\hline I think about cattle feelings when I euthanize it & 1 & 1 & 1.5 & 2 & 0.08 \\
\hline If I had the choice I prefer I euthanize cattle rather than someone else & $5^{\mathrm{bc}}$ & $2^{\mathrm{a}}$ & $1.5^{\mathrm{ac}}$ & $4^{\mathrm{b}}$ & 0.05 \\
\hline My coworkers do not wait longer than they should before euthanizing cattle & $4.5^{\mathrm{c}}$ & $2^{\mathrm{bc}}$ & $2^{\mathrm{abc}}$ & $4^{\mathrm{a}}$ & 0.04 \\
\hline Job knowledge & 5 & 5 & 4 & 5 & 0.10 \\
\hline The best place to keep an electric prod is away from me & 4 & 2 & 2 & 2 & 0.30 \\
\hline I am good at handling cattle & 5 & 5 & 4.5 & 5 & 0.17 \\
\hline I have enough knowledge to know what to do with sick or injured cattle & 5 & 5 & 4 & 5 & 0.06 \\
\hline It is easy to identify sick or injured cattle & 5 & 4 & 4 & 5 & 0.34 \\
\hline I feel confident providing medical treatment to sick cattle & 5 & 5 & 5 & 5 & 0.19 \\
\hline
\end{tabular}


Table 4: Multiple choice questions and responses provided exclusively from pen riders

\begin{tabular}{lc}
\hline Question & Results $(\%)$ \\
\hline How many pens do you ride in a day? & 50 \\
Less than 20 & 30 \\
10 to 20 pens & 30 \\
20 to 30 pens & 84 \\
$30+$ pens & \\
What method do you use to ride pens? & 32 \\
Waves & 51 \\
Sections & 80 \\
Both & 50 \\
Other & \\
\hline
\end{tabular}

\section{Discussion}

Survey results showed that Texas beef cattle stock people possess a high level of job satisfaction, believe themselves to hold adequate job knowledge and seem to trust and communicate with their coworkers. Stock people differed by role regarding animal perception, confidence in performing euthanasia and aspects of their daily workload. Regardless of role, all stock people displayed a negative bias towards dairy cattle in the feed yard.

\section{Differing Views Regarding Euthanasia}

Differing perceptions among employees in different departments within the feed yard regarding timely euthanasia could contribute to a lack of continuity in animal treatment and on-farm protocols, and may influence the overall workplace culture, as disagreements about when euthanasia should be performed may create conflict among employees. Pen riders were reportedly less confident in performing euthanasia than doctors and management, potentially because they are less likely to perform euthanasia relative to the other roles. Pen riders reported lower levels of confidence in their coworkers' ability to make timely decisions about when an animal should be euthanized. Considering pen riders are the stewards of cattle health and directly interact with the cattle on a daily basis, their reported lack of confidence in performing euthanasia, combined with differing opinions among coworkers about when euthanasia is performed, indicates that continuing education regarding end-of-life decisions is needed and that additional training tools would benefit the employees and the animals.

Knowledge of how and when to perform euthanasia, confidence in performing euthanasia, and empathy all effect stockperson perception of performing and coping with euthanasia on farm (Rault et al., 2017). In the swine industry, the physical capacity to perform euthanasia varied at the individual level (Widowski et al., 2008) and can be influenced by the confidence level of the individual stockperson performing the euthanasia. Stock people with less confidence in performing euthanasia were reportedly uncomfortable with the concept overall. Employees that had more difficulty deciding when to euthanize had less knowledge regarding how to perform euthanasia, suggesting that providing adequate training designed to increase knowledge and confidence regarding euthanasia could promote positive perceptions of euthanasia in the animal industry and minimize animal suffering (Rault et al., 2017). These results illustrate that euthanasia protocol education for all employees that will be required to perform euthanasia and make end-of-life decisions is beneficial. All feed yard employment roles can benefit from end-of-life training as part of an effort to promote a positive animal welfare culture among feed yard employees and to propagate positive perceptions regarding on farm euthanasia.

\section{Happy, but Overworked and Underpaid}

Participants had high levels of job knowledge (excluding timing of euthanasia), communication, and trust regardless of role. However, irrespective of these high levels of knowledge and communication, issues with animal care may still be present. Wambui et al. (2018) observed that, while Kenyan cattle stock people demonstrated high levels knowledge and positive attitudes toward animals, their animal handling practices were poor. This survey highlighted a disconnect among education, job satisfaction, and employee behavior. Understanding the relationship among these different factors provides feedback as to what information should be included in new industry training protocols. As animal handling is a skill that requires training and practice, active learning and hands-on training may be effective in communicating important concepts regarding animal handling to stock people. Integrating hands-on training to supplement the standard classroom-learning environment may reinforce and further develop a stockperson's skills throughout the whole duration of their career.

Providing satisfactory on-the-job training can promote job satisfaction through increasing job 
knowledge (Schmidt, 2007) and can increase overall workplace well-being, which is essential to job satisfaction (Biggie and Cortes, 2013). By providing interactive and dynamic training, Texas feed yard operators can improve stockperson perceptions and behaviors, improve the HAI on farm and cultivate positive attitudes in the work environment. Through providing workplace-focused behavioral training to swine handlers, stock people viewed pigs in a more positive manner and pigs displayed less fear behaviors towards those stock people that had undergone training (Hemsworth et al., 1994; Coleman et al., 2000). Coleman et al. (2000) also concluded that swine stock people who have received training display higher levels of job retention. This research suggests that the initial benefits from providing stockperson training are tangible and providing targeted training can improve the HAI.

While the survey highlighted the positive workplace culture in Texas feed yards, a variety of socio-economic challenges were identified. Although there was substantial variety in employee education level, age, and language fluency, the survey results depicted a population of experienced and well-intentioned employees. The majority of the participants have been in the industry for over 6 years and were choosing to work in the industry because they were dedicated to animal care. Educational requirements for stock people are changing as more feed yard employees are becoming college educated.

Youth raised in urban settings are gaining agriculture knowledge exclusively in classroom setting. Subsequently, a generational gap in knowledge acquisition is forming between the younger and the older, more experienced stock people, who were likely trained using hands-on, on-the-job training, or through being raised in an agricultural setting (Daigle and Ridge, 2018). However, despite the high level of experience and the increasing educational requirements, most participants are working well over $40 \mathrm{hrs} / \mathrm{wk}$ for less than $\$ 15 / \mathrm{hr}$.

According to the USBLS (2018b), annual nonmanagerial stockperson salary $(\$ 25,470)$ is less than $\$ 1,000$ above the national poverty line for a 4-person household in the United States $(\$ 24,848)$. Considering the survey results report that over half of the participants served as the sole source of income for their whole household, the low level of pay should be concerning from both a monetary and "employee retention" perspective. Relative to other occupations that interact with animals on a daily basis, stock people receive the lowest compensations, regardless of working long hours in adverse conditions (Daigle and Ridge, 2018).

The survey also provided evidence that beef cattle stock people are overworked. Increased urbanization, fewer trained stock people in the livestock industry and the unrealistic work expectations of stock people are contributing to an overworked employee base in the industry (Daigle and Ridge, 2018). The average employed American works about $40.3 \mathrm{hrs} / \mathrm{wk}$ (USBLS, $2018 \mathrm{c}$ ), yet $76 \%$ of survey feed yard employee participants work over $50 \mathrm{hrs} / \mathrm{wk}$. Stock people work an average of $54.9 \mathrm{hrs} / \mathrm{wk}$ (Jensen and Mark, 2010) and these long hours can have detrimental consequences to cattle welfare and beef sustainability, including employee fatigue, poor decision making, improper animal handling, or higher employee turnover (Daigle and Ridge, 2018). Alonso and colleagues (2017) highlighted that working over $48 \mathrm{hrs} / \mathrm{wk}$ increases burnout, puts employees at a higher risk of anxiety and depression and reduces sleep time. Regulating the workload and increasing the pay of stock people and feed yard employees is needed to retain good stock people, provide employees with a living wage, promote positive public perception of agricultural animal stock people and maintain positive on-farm cattle welfare and agricultural sustainability in the United States.

\section{Perception of Cattle and Breed Biases}

In this survey, stock people tended to view cattle in a negative manner regarding their cleanliness. The phrase "cattle are dirty" could have been interpreted differently at the individual stock person level, influencing the results. Although these results do not indicate a direct dislike of animals, this does suggest there is potential for negative HAI on farm. Stock people in the swine industry who viewed pigs as "dirty" and "glutinous" were more likely to have negative interactions (e.g. forceful slaps) with the pigs in their care (Coleman and Hemsworth, 2014). The internal perceptions and attitudes of stock people motivate their external actions towards animals and have the potential to influence the valence of the human-animal relationship (Coleman and Hemsworth, 2014). Understanding a stockperson's attitude and perception may provide insight into the type of HAI that may be occurring between the stockperson and the cattle in their care.

A majority of cattle stock people view Holstein cattle unfavorably. This is a concern considering Holsteins fed in commercial feed yards account for approximately $15 \%$ of the marketed fed cattle on the United States (QueckMatzie, 2019). Although research has not been conducted on Holsteins in a feed yard, substantial research has evaluated the impact of the HAI on Holsteins housed in the dairy industry. For example, stock people within the dairy industry possessing positive attitudes toward animals displayed higher levels of patience when moving and milking cows and were more willing to have physical contact with cows (e.g., hands-on cow grooming). Alternatively, the same study observed that dairy stock people with negative attitudes towards cows were more likely to punish (e.g., hit) cows while milking (Waiblinger et al., 2002). In swine abattoirs, handlers with negative attitudes toward pigs tended to use 
the electric prod more frequency when moving pigs compared to those who viewed swine in more positive manner (Coleman et al., 2003). In commercial swine operations, stock people who believed in petting pigs and using minimal force and noise to move pigs tended to have fewer negative beliefs about pigs (Hemsworth and Coleman, 2011). Therefore, the dislike of certain cattle types by Texas stock people suggests that Holstein cattle may be treated differently than traditional beef breeds.

Surveyed pen riders and processors were more likely is disagree that "cattle behavior is not effected by the way we treat them", identifying that the stock people that have the most frequent and intense interactions with cattle may not understand the importance of their actions to cattle welfare, especially their ability to induce a fear response. Fear is physiologically evident through increased, long-term release of corticosteroids and can be behaviorally quantified by avoidance behaviors (Hemsworth and Coleman, 2011). Pigs observed on a commercial swine operation receiving inconstant and unpleasant interactions with handlers had increased blood corticosteroid concentrations and displayed more avoidance behaviors than pigs that experienced minimal and pleasant interactions with humans (Hemsworth et al., 1987).

The fear response induced from negative handling compromises not only welfare, but also productivity and product quality. Dairy cows that received more negative tactile interactions had significantly decreased milk protein and fat, and cattle handled at faster handling speeds exhibited decreased milk yields (Breuer et al., 2000). In beef cattle, distressful HAI has been observed to negatively impact cow reproductive success. Macedon et al. (2011) observed that when performing embryo retrieval from Bos indicus beef cows, individual animals that experienced intense, negative handling situations prior to embryo retrieval produced $19 \%$ fewer viable embryos than animals that experienced positive stockperson interactions. A lack of stockperson understanding that human behaviors effects animal welfare signals a gap in knowledge in HAI. All stock people may not be aware of how their actions impact the animal's fear response and subsequent productivity of food animals. This emphasizes the importance of educating employees about the HAI during training and that continuing education is critical to employee performance and overall sustainability (Daigle and Ridge, 2018). Empirical evaluation of how stockperson perception of cattle affects handling is needed to elucidate the strength and severity of this relationship with regards to beef cattle.

\section{Implications for Feedlot Cattle Welfare and Beef Sustainability}

Each employee at the feed yard, irrespective of role, has an impact on cattle welfare. Feed truck drivers and feed mill workers contribute to cattle welfare through the provision and distribution of properly prepared food while yard crew employees ensure a safe and comfortable environment and fresh water. Pen riders and doctors develop a positive human-animal relationship and maintain good animal health across the feed yard. Processing crews administer vaccinations and, as the first feed yard employees to interact with incoming cattle, play a role in establishing that the feed yard is a safe and comfortable place for cattle to live. Managers influence cattle welfare through the choices they make regarding cattle management and employees actions.

Strikingly, no yard crew employees and very few feed truck drivers participated in the training or survey. Feed truck drivers should be trained in cattle behavior as they regularly see the cattle from a non-threatening perspective (from a feed truck) performing behaviors that provide pleasure. Therefore, cattle will be less likely to mask injury or sickness as part of the prey survival instinct. Yard crews manage cattle environments and keep water tanks clean, directly impacting welfare. These employees have regular and direct interactions with cattle throughout the day, thus the type of interactions these employees have with the cattle may be different compared to pen riders and their actions can influence welfare, cattle behavior and profitability. Future educational efforts should strive to include all employees at the feed yard, as those that are not engaged in continuing education may have a substantial impact on cattle welfare and feed yard profitability.

Survey results also highlighted potential difficulties that may be faced by the Texas beef industry in the near future. As the population of the United States becomes increasingly urban, securing the next generation of animal-agricultural employees will prove to be a challenge (Daigle and Ridge, 2018). Gaps in age, education and language will only expand over time. Results show that $38 \%$ of the survey participants were over 40 years of age. This dedicated subgroup of the workforce will begin aging out of feed yard work within the next two decades, creating a large demand for employees in the animal agriculture field. The workforce is diverse not only in age, but in educational background. While $54 \%$ of participants have less than or equal to a high school education, as the methods of production evolve and precision agriculture becomes more mainstream, the demand for college educated employees is may increase (USBLS, 2018a). The industry must adapt to these changes to ensure longterm workforce sustainability and to create workplace environments that foster positive animal welfare.

Differing education levels and the addition of Spanish as a primary dialect in the stockperson 
community creates a diverse and changing workforce. Effective communication is paramount to the efficient use of time, resources, and transfer of knowledge. Lack of communication can influence veterinary treatments and animal health status updates, both of which compromise welfare and productivity. Identifying strategies that facilitate effective communication among employees, without burdening those that serve as translators, would benefit the cattle and the employees.

\section{Conclusion}

Moving forward, focus must be placed on improving and maintaining the employee perception towards animals to ensure that all animals are being handled in a manner that minimizes fear and fosters a positive human-animal relationship. All stock people, irrespective of role, must increase their level of comfort regarding euthanasia techniques and protocols in effort to provide ethical lifeending decisions for cattle. Initial and continuing education that combines hands-on learning with classroom style teaching and emphasizes the importance of the stockperson to cattle welfare is critical for improving stockperson attitudes and creating a long-term educated workforce. Investing in employees by providing training that is engaging and caters to the areas of improvement identified by this survey may not only increase animal welfare knowledge but also increase job satisfaction and workplace morale.

The socio-economic results of the survey highlight that stock people are overworked and underpaid, which undermines any effort to encourage people outside the realm of animal agriculture to consider a career as a cattle stock person. Workload and pay must be adjusted to reflect physical and mental demands of the job as well as the strength of impact stock people can have on the productivity, profitability and social acceptability of animal agriculture. The beef cattle industry must prepare for these impending workforce changes, as younger and more college-educated stock people enter the field. The increasing gaps in language and age will provide a hurdle for Texas feed yard employers, but adaptation is necessary to maintain a dedicated workforce. Through expressing gratitude and placing value on this diverse workforce, the industry can increase animal welfare and promote longterm sustainability of beef cattle production.

\section{Acknowledgments}

The authors would like to thank the Texas Cattle Feeders Association for allowing us to administer the survey at their event; also the Statistical Computing Center at Teas A\&M University, College Station for their assistance in the statistical analysis of the results.
Thank you to Dr. Ted McCollum for his support of this project. Additionally, thank you to Michelle CalvoLorenzo and Hector Levy-Jimenez for translating the survey from English to Spanish.

\section{Author Contributions}

Survey creation, data exploration and article construction was conducted as a joint effort of Courtney L. Daigle and Emily E. Ridge. Ron Gill established industry connection as well as aided in survey development.

Emily. E. Ridge: Survey development, data collection and analysis, manuscript preparation

Ron Gill: Survey development, manuscript preparation

Courtney L. Daigle: Survey development, data collection and analysis, manuscript preparation.

\section{Conflict of Interest}

There is no conflict of interest associated with this study.

\section{References}

Alonso, P., M. Fonesca and J.F. Pires, 2017. Impact of working hours on sleep and mental health. Occupant Med., 67: 377-382. DOI: 10.1093/occmed/kqx054

BCO, 2015. Beef Quality Assurance. Cattle Care and Handling Guidelines. National Cattlemen's Beef

Biggie, G. and C.G. Cortes, 2013. Well-being in the workplace through interaction between individual characteristics and organizational context. Int. J. Qualitative Stud. Health Well Be, 8: 1-13. DOI: 10.3402/qhw.v8i0.19823

Breuer, K., H.P.H. Worth, J.L. B. Arnett, L.R. Matthews and G.J. Coleman, 2000. Behavioral response to humans and the productivity of commercial dairy cows. Applied Anim. Behavior Sci., 66: 273-288. DOI: 10.1016/S0168-1591(99)00097-0

Carlstead, K., S. Paris and J.L. Brown, 2019. Good keeper-elephant relationships in North American zoos are mutually beneficial to welfare. Applied Animal Behav. Sci., 211: 103-111. DOI: $10.1016 /$ j.applanim.2018.11.003

Coleman, G.J., P.H. Hemsworth and M. Hay, 1998. Predicting stockperson behaviour towards pigs from attitudinal and job-related variables and empathy. Applied Anim. Behavior Sci., 58: 63-75. DOI: 10.1016/S0168-1591(96)01168-9

Coleman, G.J. and P.H. Hemsworth, 2014. Training to improve stockperson beliefs and behavior towards livestock enhances welfare and productivity. Rev. Sci. Tech. Off. Int. Epiz., 33: 131-137. DOI: $10.20506 /$ rst.33.1.2257 
Coleman, G.J., M. McGregor, P.H. Hemsworth, J. Boyce and S. Dowling, 2003. The relationship between beliefs, attitudes and observed behaviors of abattoir personnel in the pig industry. Applied Anim. Behavior Sci., 82: 189-200. DOI: 10.1016/S0168-1591(03)00057-1

Coleman, G.J., P.H. Hemsworth, M. Hay and M. Cox, 2000. Modifying stockperson attitudes and behavior toward pigs at a large commercial farm. Applied Anim. Behavior Sci., 66: 11-20. DOI: 10.1016/S0168-1591(99)00073-8

Daigle, C.L. and E.E. Ridge, 2018. Investing in stockpeople is an investment in animal welfare and agricultural sustainability. Anim. Frontiers, 8: 53-59. DOI: $10.1093 /$ af/vfy015

Rault, J.L., T. Holyoake and G. Coleman, 2017. Stockperson attitudes toward pig euthanasia. J. Animal Sci., 95: 949-957. DOI: $10.2527 /$ jas.2016.0922

Hemsworth, P.H., G.J. Coleman, J.L. Barnett, S. Borg and S. Dowling, 2002. The effects of cognitive behavioral intervention on the attitude and behavior of stockpersons and the Behavior and productivity of commercial dairy cows. J. Anim. Sci., 80: 68-78. DOI: $10.2527 / 2002.80168 x$

Hemsworth, P.H., J.L. Barnett and C. Hansen, 1987. The influence of inconsistent handling by humans on the behaviour, growth and corticosteroids of young pigs. Applied Anim. Behavior Sci., 17: 245-252. DOI: $10.1016 / 0168-1591(87) 90149-3$

Hemsworth, P.H. and J.L. Barnett, 1989. Relationship between fear of humans, productivity and cage position of laying hens. British Poultry Sci., 30: 505-18. DOI: 10.1080/00071668908417175

Hemsworth, P.H., G.J. Coleman and J.L. Barnett, 1994. Improving the attitude and behavior of stock people towards pigs and the consequences on the behavior and reproductive Performance of commercial pigs. Applied Anim. Behavior Sci., 39: 349-362.

DOI: 10.1016/0168-1591(94)90168-6

Hemsworth, P.H., J.L. Barnett and G. J. Coleman, 1993. Improving productivity with better stocking handling. In Proceedings of the New Zealand Society of Anim. Produc., 53: 211-213.

DOI: $10.1093 /$ occmed/kqx054

Hemsworth, P.H. and G.J. Coleman, 2011. HumanLivestock Interactions: The Stockperson and the Productivity and Welfare of Intensively Farmed Animals. 1st Edn., CABI, Wallingford, UK, ISBN-10: 1845936736, pp: 194.

Janzen, I., 1991. The theory of planned behavior. Organized. Behavior Human Decius. Processes, 50: 179-211. DOI: 10.1016/0749-5978(91)90020-T

Jensen, R. and D.R. Mark, 2010. Nebraska feed yard labor Cost benchmarks and historical trends. Department of Agricultural Economics, University of Nebraska.
.Macedon, G.G., C.E. Serra Nett Zackary, U.G. Pinto de Abreu, J.A Begrao and E.V. Costa de Silva, 2011. Human-animal interactions, stress and embryo production in boss indies embryo donors under tropical conditions. Tropical Anim. Heal. Produce., 43: 1175-1182. DOI: $10.1007 / \mathrm{s} 11250-011-9820-6$

Queck-Matzie, T., 2019. Consumer demand drives fed Holstein market. Feedlot Magazine.

R. Core Team, 2018. R: A language and environment for statistical computing. R Foundation for Statistical Computing, Vienna, Austria.

Russia, S., 2003. Human-cattle interaction on group housing. Applied Anim. Behav. Sci., 80: 245-262. DOI: 10.1016/S0168-1591(02)00213-7

Sari, L.M., and Judge, T.A. 2004. Employee attitudes and job satisfaction. Human Resource Management 43:4, 395-407. DOI: 10.1002/hrm.20032

Schmidt, S.W., 2007. The relationship between satisfaction with workplace training and overall job satisfaction. Human Res. Develop. Quarterly, 18: 481-498. DOI: $10.1002 / \mathrm{hrdq} .1216$

Tucker, C.B., J.F. Coetzee, J.M. Stookey, D.U. Thomson and T. Grandin, et al., 2015. Beef cattle welfare in the USA: Identification of priorities for future research. Anim. Heal. Res. Rev., 16: 107-124. DOI: $10.1017 / \mathrm{S} 1466252315000171$

USBLS, 2018a. How to become a farmer, ranchers or other agricultural manager. United States Bureau of Labor Statistics.

USBLS, 2018b. Occupational employment and wages: Farm workers, farm, ranch and aquacultural animals. United States Bureau of Labor Statistics.

USBLS, 2018c. Average hours employed people spent working on days worked by day of week. United States Bureau of Labor Statistics.

Waiblinger, S., C. Menke and G. Coleman., 2002. The relationship between attitudes, personal characteristics and behavior of stock people and subsequent behavior and production of dairy cows. Applied Anim. Behavior Sci., 79: 59-219. DOI: 10.1016/S0168-1591(02)00155-7

Wambui, J., P. Lamuka, E. Karuri and J. Matofaru, 2018. Animal Welfare Knowledge, Attitudes and Practices of Stockpersons in Kenya. Anthrozoos, 31: 397-410. DOI: $10.1080 / 08927936.2018 .1482111$

Widowski, T.M., R.H. Elgie and P. Lewis, 2008. Assessing the effectiveness of a non-penetrating captive bolt for the euthanasia of newborn piglets. Proceedings at Allen C. Leman Swine Conference, (LSC' 08), pp: 107-111. 